Results In total, 310 participants were included in the study (response $70 \%$ ). The majority were men who have sex with men. Seventy five percent of the study population was referred directly from STI clinic to treatment centre by a clinician or nurse. Thirty percent of participants were delayed: $15 \%$ entered care after 4 weeks and $15 \%$ had not entered care yet. Thus, $70 \%$ of participants entered care within 4 weeks; median time of entry was 9 days (range 0-739 days). In the multivariate model, age below 25 years ( $\mathrm{OR}=3.695 \%$ CI 1.9-6.7) and indirect referral (OR $=3.395 \%$ CI 1.8-6.5) were independent risk factors for care delay, while people with health insurance were less likely to delay ( $\mathrm{OR}=0.395 \%$ CI $0.1-0.9)$. No association was found between care delay and CD4 cell counts or viral load at diagnosis.

Conclusions Younger persons and persons without health insurance are at risk for entering care late after being diagnosed HIV positive. Direct referral from STI clinic to care leads to less delay. Testing of those at risk is not enough to interrupt HIV transmission, entry into care needs to be assured as well. A more active role of the care provider is needed.

\section{P2.154 DETERMINANTS OF UTILIZATION OF A NO-COST HIV TRANSITION CLINIC IN UGANDA: A CROSS SECTIONAL STUDY OF YOUNG ADULTS LIVING WITH HIV/AIDS}

doi:10.1136/sextrans-2013-051184.0418

A Nyabigambo. Makerere University, College of Health Sciences, School of Public Health, Kampala, Uganda

Objective The main purpose of this study was to understand the levels and determinants the HIV Transition Clinic services utilisation by young adults at Infectious Diseases Institute, Kampala, Uganda.

Methods A cross sectional study using quantitative methods at a HTC in a sample of 379 young adults living with HIV/AIDS (YALHA) between the ages of 15-24 years. At analysis utilisation was categorised into two levels; regular (kept all appointment visits) and irregular (missed one or more appointments visits) utilisation. Univariable, bivariable and multivariable logistic regression was used to establish determinants associated with utilisation of the HTC.

Results Of the 379 total respondents, only $32.4 \%$ were regular utilizers of the HTC. There are low levels of regular utilisation of the HTC. Female young adults have better service utilisation rates compared to the males in HTC. The male to female ratio was 1:5. The determinants of HTC regular utilisation were CD4 cell count category of 250-2603/ $\mu \mathrm{l}$ (AOR 0.58, 95\% CI: 0.36-0.95), not currently on ART (AOR 0.27, 95\% CI: 0.15-0.47) and did not receive counselling services (AOR 0.47, 95\% CI: 0.27-0.83).

Conclusion The factors that were associated with reduced the chance of regular use of the HTC were: CD4 cell count between 250-2603/ $\mu \mathrm{l}$; not being on ART; and not receiving HIV counselling services.

Recommendations The young adults in the HIV transition clinic should be screened on a regular basis to detect those with a CD4 cell count of $<350 / \mu \mathrm{l}$ and counselled for early initiation of ART so as to enhance regular HIV transition clinic utilisation. In addition a reminder system like peers, family and mobile phone messages should be set up for young adults so that they are reminded of their appointments hence facilitating regular use of the transition clinic.

\section{P2.155 ADHERENCE MATTERS: A PATIENT-CENTERED ADHERENCE STRATEGY TO ACHIEVE MAXIMUM HEALTH AND QUALITY OF LIFE OUTCOMES FOR PERSONS LIVING WITH HIV/AIDS IN PLAINFIELD, NEW JERSEY}

doi:10.1136/sextrans-2013-051184.0419

L Kudryashova Hernandez. Neighborhood Health Services Corporation, Plainfield, NJ, United States
Background Neighborhood Health Services Corporation (NHSC), an urban community-based not-for-profit ambulatory primary care health centre located in Plainfield, New Jersey, USA, provides HIV care and treatment services to over 350 uninsured, impoverished and minority persons living with HIV/AIDS. To achieve long-term positive health and quality of life outcomes, it is imperative that patients adhere to HIV care and treatment, as well as to prescribed medication regimens.

Methods At NHSC patient-centred adherence efforts begin with a personalised interview. An assessment of patient's clinical and lifestyle indicators is conducted by clinicians followed by formal adherence education video presentations. Other key components of the adherence strategy are detailed discussions of antiretroviral therapies and potential side effects. Patients are provided with culturally and linguistically sensitive printed materials regarding medications and potential side effects. Free Medication boxes are also provided. Weekly monitoring phone calls are conducted by clinical staff to assess compliance and identify adherence challenges. If patients miss appointments, confidential phone calls are placed to reschedule. Treatment Education and Adherence Record is a comprehensive tool that is administered annually to assess adherence, compliance, barriers and any unanticipated outcomes. A multi-disciplinary clinical team led by an Infectious Disease Specialist observes the entire process and monitors patients' boosted compliance with the project and promotion of overall HIV treatment and medications adherence.

Results Resulting from the patient-centred adherence strategy, NHSC observed: $25 \%$ improvement in medical visits no-show rates; $10 \%$ decrease in hospitalisation rates. Furthermore, $62 \%$ of patients maintained CD4 above 200; $52 \%$ of patients have achieved and maintained undetectable viral load.

Conclusions The collected and analysed data suggests that patient-centred adherence efforts help to: reduce no-show rates; minimise side effects and hospitalizations related to missed doses and "drug holidays"; improve and sustain optimal clinical indicators, as well as significantly improve health and quality of life outcomes.

\section{P2.156 PLACE OF NURSES IN THE MANAGEMENT OF PEOPLE LIVING WITH HIV AND AIDS (PLHA)IN DEVELOPING COUNTRIES: CASE OF A REGIONAL HOSPITAL, BURKINA FASO (WEST AFRICA)}

doi:10.1136/sextrans-2013-051184.0420

'Y Sagna, ${ }^{2} \mathrm{M}$ Mano, ${ }^{1,3} \mathrm{~J}$ Y Drabo. ${ }^{1}$ Internal Medicine Department of Yalgado Ouédraogo University Hospital (CHU YO), Ouagadougou, Burkina Faso; ${ }^{2}$ Medicine Department of Tenkodogo Regional Hospital, Tenkodogo, Burkina Faso; ${ }^{3}$ African network of training on HIV (RAF-VIH), Ouagadougou, Burkina Faso

Background Management of people living with HIV and AIDS (PLHA) in the Tenkodogo regional hospital was carried out by a team of 7 nurses and 4 physicians engaged also about their function in the management of non-HIV-infected patients. Only one of these nurses had already received specific training (inter-university graduate) on the management of HIV infection. This team had to manage actually 1213 PLHA including 505 under antiretroviral treatment (ART). Objective We aimed to describe the activities carried out by nurses of this team in the management of PLHA.

Methods We retrospectively reviewed all consultation activities for PLHA by the 7 nurses of the HIV-infected patient's management team at the Tenkodogo regional hospital from January to June 2011. The daily consultation registers of these nurses were our data sources.

Results 2709 consultations of PLHA (any reason confused) were carried out. The consultations mean was 451.5/month. 1886 ART renewals prescription have been made, including 1767 first line and 119 second line treatment. 44 PLHA were newly treated by ART 